\title{
A Quality-Based Birth-and-Death Queueing Model for Evaluating the Performance of an Integrated Voice/Data CDMA Cellular System ${ }^{1}$
}

\author{
Chyi-Nan Wu* , Ywh-Ren Tsai** and Jin-Fu Chang* \\ *Electrical Engineering Department, National Taiwan University, Taipei, Taiwan 10764 \\ **Transmission Technology Laboratory, Telecommunication Laboratories, Taoyuan, Taiwan 326
}

\begin{abstract}
CDMA has been proven to be one of the promising mainstream multiaccessing techniques in future cellular mobile communication systems. It is offering attractions such as soft capacity, soft handoff, antimultipathing, and etc. In this paper a quality-based birth-and-death queueing model is developed for the purpose of evaluating the performance of a CDMA cellular that supports two-rate transmissions.
\end{abstract}

\section{INTRODUCTION}

Mobile communication or wireless communication has recently become one of the hottest research topics. Cellular phones have closely linked with many people's daily lives. The subscription to mobile phone service in many countries has increased drastically in recent years. Predicition of business opportunities in wireless communication has been overwhelmingly good. PCS (Personal Communication Service) and PCN (Personal Communication Network) are two well understood acronyms to many technical and business people.

The technology of wireless communication has advanced from the first generation of analog systems to the new generation of digital systems. AMPS (Advanced Mobile Phone Service) [1] is a well known and widely implemented analog cellular system and supports primarily voice communications. Analog systems have the problems such as capacity limitation, less noise/interference immunity, security loophole, $\cdots$. Digital technology overcomes many of these problems. It also has a very important selling point of being able to easily support a wide variety of applications ranging from the traditional voice to new applications such as data, fax, message, video and etc. European GSM (Global System for Mobile communications), North American IS-54 and Japanese PDC (Personal Digital Cellular) are three examples of digital cellular systems [2]. They are also examples of TDMA (Time Division Multiple Access) systems.

CDMA (Code Division Multiple Access) is another mainstream and competing (access) technique in implementing a digital cellular system, e.g. [3]. CDMA is usually realized through spread spectrum (SS) techniques [4], either direct sequence (DS) or frequency hopping (FH), or a hybrid between DS and FH. CDMA has been reported to offer attractions such as more

${ }^{1}$ The work reported in this paper is aupported by the National Science Council, Rep. of Chine under Grant NS C-84-2221-E-002-037 (1995).

0-7803-3002-1/95\$4.00 C1995 IEEE efficient spectral utilization, soft capacity, soft handoff, antimultipath fading [5]-[7]. It also has a unique feature of offering the flexibility of letting CDMA users share spectrum with some existing microwave users without disrupting their service [8]-[9].

In cellular systems, each cell receives two types of call : new calls and handoff calls. CDMA cellular is no exception. So far there has been very limited study done on the construction of appropriate queueing model for evaluating the performance of a CDMA cellular system. The purpose of our work is to establish such queueing model. The model shall not only take both types of call but also the soft capacity feature of CDMA cellular into account. It is widely known that the quality of CDMA system degrades gradually not abruptly as more calls are admitted. Calls are rejected only when the quality of service becomes untolerable. In handling whether to admit or reject a call, priority may be given to favor handoff calls in order to reduce the drop rate. The model constructed in our paper shall allow such flexibility.

Although CDMA is not the only solution to support multirate service, it is indeed a good candidate technique. In the future CDMA system, a good variety of narrow band and broad band applications is expected to be supported. But at the early stage of deployment it is quite possible that only voice and primitive type of data are to be experimented. Because of this consideration we shall in this paper concentratie on the modeling of a dual rate CDMA system.

In CDMA environment, the quality of transmission on the reverse, i.e. mobile to base station, link is susceptible to the well known near-far effect. The power radiated from a base station may overflow into adjacent cells to interfere the forward, i.e. base station to mobile, link transmission in these adjacent cells. Power control [5]-[6], [10] are exercised on both forward and reverse link to reduce such effects. The work in this paper is to be done under the assumption of perfect power control.

Let $r_{j}$ denote the distance between a mobile, say $j$, and its base station. Let $R$ denote the radius of a general cell. We use $P_{r}\left(r_{j}\right)$ and $P_{f}\left(r_{j}\right)$ to denote the power transmitted from mobile $j$ (to its base station) and the power transmitted to mobile $j$ (from its base station), respectively. We also use $P_{T}$ and $P_{T}^{\prime}$ to de- 
note the maximal power possibly transmitted from a mobile at cell boundary and the maximal power possibly transmitted to reach a mobile near cell boundary. Under the assumption of perfect power control

$$
\begin{aligned}
& P_{r}\left(r_{j}\right)=P_{T}\left(\frac{r_{j}}{R}\right)^{4} \\
& P_{f}\left(r_{j}\right)= \begin{cases}P_{T}^{\prime}\left(\frac{0.6 R}{R}\right)^{2}, & r_{j} \leq 0.6 R \\
P_{T}^{\prime}\left(\frac{r_{j}}{R}\right)^{2}, & r_{j}>0.6 R\end{cases}
\end{aligned}
$$

The purpose of power control is to let the base station receive equal carrier-to-interference ratio $(C / I)$ from all mobiles and vice versa. Eq. (2) is taken from [10].

Suppose a cell currently contains a total of $M$ mobiles. Like other studies we assume that these mobiles are distributed uniformly in the cell. Let $\sigma$ denote the user density, then

$$
\sigma=\frac{M}{4 \pi R^{2}}
$$

Lee has in [5] derived the $C / I$ on the forward link for a CDMA cell under the assumption of perfect power control. But the power control law used by Lee is slightly different from the one outlined in (2). In Lee's work, $0.55 R$ is used, while $0.6 R$ is used in (2). We decide to adopt $0.6 R$ because it is obtained from simulation and somehow more emperical. Modify Lee's result to fit (2), we obtain

$$
\frac{C}{I}=\frac{1}{1.8695 M-1}
$$

Let us consider a CDMA system which uses BPSK for modulation. Let $\boldsymbol{R}_{b}$ denote the source's bit rate and $W$ the bandwidth occupied by the CDMA system. Using $P_{e}$ to denote bit error rate of the system, then [11]

$$
P_{e}=\frac{1}{2} \operatorname{erfc}\left(\sqrt{\frac{C / I}{R_{b} / W}}\right)
$$

where erfc denotes the well-known complementary error function

$$
\operatorname{erf}(x)=\frac{2}{\sqrt{\pi}} \int_{x}^{\infty} e^{-t^{2}} d t
$$

Use (4) and (5) and let $W=1.25 \mathrm{MHz}, R_{b}=8.0$ kbps we may conclude that a cell can accommodate $M=17 R_{b}$-bps users if the required $P_{e}$ is set at $10^{-3}$ and $M=27$ if $P_{e}$ is relaxed to $5.0 \times 10^{-3}$. Notice that in the capacity calculation here we exclude the consideration of voice activity and sectorization.

There is concern that data is error sensitive and $10^{-3}$ is not enough for data transmission. Forward error correction plus retransmission must be used to achieve the bit error rate requirement for data users. This also partially tells why our data transmission is assumed to take up a bandwidth $b$ times of a voice communication.

Consider no more than a total of 18 interferring neighboring cells, We have obtained for the reverse link $^{2}$

$$
\begin{aligned}
& I \cong \\
& \quad \frac{\alpha(M-1) P_{T}}{R^{4}}+\frac{\alpha M P_{x}}{R^{4}}[6 f(\sqrt{3})+6 f(3)+6 f(2 \sqrt{3})]
\end{aligned}
$$

where $\alpha$ is the constant of proportionality in the 40 $\mathrm{dB} /$ dec propagation path loss model and

$$
f(x)=\frac{4 x^{2}-3 x^{4}}{\left(x^{2}-1\right)^{2}}-4 x^{2} \ln \left(1-\frac{1}{x^{2}}\right)-1
$$

Let $\boldsymbol{P}_{c}$ denote the power received by a base station from a mobile, say $j$, at distance $r_{j}$, then under the assumption of perfect power control

$$
P_{c}=\alpha P_{T}\left(\frac{1}{R}\right)^{4}
$$

Thus on the reverse link

$$
\begin{aligned}
\frac{C}{I} & =\frac{P_{c}}{I} \\
& =\frac{1}{M[1+6 f(\sqrt{3})+6 f(3)+6 f(2 \sqrt{3})]-1}(8)
\end{aligned}
$$

From (5) and (8) we obtain for the reverse link when $P_{e}$ is desired at $10^{-3}$ the system can support $M=18$ users transmitting at the speed $R_{b}$ bps and if $P_{e}$ is relaxed to $5.0 \times 10^{-3}$ the channel capacity can be increased to 29. Compare with forward link, we observe that reverse link gives slightly better capacity. This difference is also observed in Lee's work [5]. In Lee's reverse link capacity analysis, interference from neighboring cells is not included in the evaluation of $\mathrm{C} / \mathrm{I}$. Here, we take the interference from neighboring cells into consideration.

The rest of this paper is organized as follows. In Section II we establish a birth-death queueing model for the derivation of call blocking rate. The birth-anddeath queueing model was also used in [13] to solve a different problem. Numerical examples and discussions are given in Section III. Conclusions are made in Section IV.

\section{Performance ANalysis via Quality-BAsed BIRTh-Death Queueing ModeL}

Base on the preparation done in Section I, we shall in this section develop a birth-and death queueing model to evaluate the performance of a CDMA cellular system which supports both voice and data applications. In determining whether to admit a new/handoff call,

\footnotetext{
${ }^{2}$ A similar result has been derived in [12]. In our case cell with circumcircle is used in considering the area of a cell while Kim uses cell with incircle.
} 
the criterion is whether the link capacity developed in Section I would be violated.

Let each data application need a bandwidth $b$ times bigger than a voice call. In other words, suppose we say a voice call consumes one channel, then to admit a data call would require the availability of a chunk of $b$ channels. In other words, data applications considered in this paper can be of wideband if $b$ is large enough.

We have in Section I divided calls into new and handoff calls. A call can be either voice or data. Let us assume that within a cell voice and data calls constitute a Poisson process with mean rate $\lambda_{v}$ and $\lambda_{d}$, respectively. Let $\lambda=\lambda_{v}+\lambda_{d}$ and $\beta=\lambda_{d} / \lambda$. Voice arrivals can be further divided into two constituent Poisson processes with mean rate $\lambda_{v, n}$ and $\lambda_{v, h}$ to represent newly lauched and handoff calls, respectively. Similarly, data arrivals are divided into two constituent Poisson processes with mean rate $\lambda_{d, n}$ and $\lambda_{d, h}$. Let $\gamma_{v}=\lambda_{v, h} / \lambda_{v, n}$ and $\gamma_{d}=\lambda_{d, h} / \lambda_{d, n}$. It is practically resonable to assume that $\gamma_{v}=\gamma_{d}=\gamma$.

We use $\left(m_{v}, m_{d}\right)$ to denote the state of a cell which represents the number of voice and data calls that are currently supported by the cell. Since a data call consumes $\boldsymbol{b} \boldsymbol{R}_{b}$-bps channels, the total amount of channel consumption corresponding to $\left(m_{v}, m_{d}\right)$ is $k=$ $m_{v}+b m_{d}$. In our earlier calculation of link zapacity wz Jiscover that for example on the forward link, a cell can support upto 17 channels when BER is set at $10^{-3}$ and this number can be pushed to 27 when BER is relaxed to $5.0 \times 10^{-3}$. These two numbers 17 and 27 are sort of bounds of the capacity region. We shall use $K_{l}$ and $K_{u}$ to denote these two bounds of the capacity region.

We said earlier that one striking feature of a CDMA system is its soft capacity. That is, admitting additional calls when link has been running at full capacity will degrade the quality of service only gradually, not abruptly. This does not occur in FDMA or TDMA system where a call definitely gets rejected when no frequency or time slot can be found available. This scenario can be modeled by shortening the remaining residence or dwell time of each individual call in a cell when the system is running beyond its link capacity. In other words, after detecting worsening quality of service, users will become impatient and tend to terminate their calls sooner.

The residence/dwell time of a mobile in a cell is taken to be exponential by some researchers, e.g. [14][15]. In this paper we shall follow their footsteps. Let $\frac{1}{\mu_{m_{v}, m_{d}}}$ be the mean dwell time of a call when a cell is running at the state $\left(m_{v}, m_{d}\right)$. Then

$$
\mu_{m_{v}, m_{\alpha}}= \begin{cases}m \mu, & 0 \leq k \leq K_{l} \\ m \mu\left(1+\xi \frac{k-K_{1}}{K_{u}-K_{l}}\right), & K_{l} \leq k \leq K_{\iota}\end{cases}
$$

Where $m=m_{v}+m_{d}, k$ has been defined to be $m_{v}+$ $b m_{d}, 1 / \mu$ is the normal mean dwell time when the cell is running under $k \leq K_{l}$, and $\xi$ is an empirical weighting factor. Larger $\xi$ yields shorter dwell time. Shortening of call dwell time is reflected in the factor $1+\xi(k-$ $\left.K_{l}\right) /\left(K_{u}-K_{l}\right)$. Notice that a feasible state $\left(m_{v}, m_{d}\right)$ must satisfy $0 \leq m_{v}+b m_{d} \leq K_{u}$.

An alternative representation of cell state is the number of channels occupied by voice and data calls, i.e. $\left(k_{v}, k_{d}\right)$ in which $k_{v}=m_{v}$ and $k_{d}=b m_{d}$. Using $\left(k_{v}, k_{d}\right),(9)$ has to be changed to

$$
\begin{aligned}
& \mu_{k_{v}, k_{d}} \\
& = \begin{cases}\left(k_{v}+\frac{k_{d}}{b}\right) \mu, & \text { if } 0 \leq k \leq K_{l} \\
\left(k_{v}+\frac{k_{d}}{b}\right) \mu\left(1+\xi \frac{k-K_{l}}{K_{u}-K_{l}}\right), & \text { if } K_{l} \leq k \leq K_{u}\end{cases}
\end{aligned}
$$

The transition between adjacent states is governed by $\lambda_{v, n}, \lambda_{v, h}, \lambda_{d, n}, \lambda_{d, h}$ and $\mu_{k_{v}, k_{d}}$ defined in (10). Clearly $\left(k_{v}, k_{d}\right)$ behaves in accordance with a two dimensional birth-and-death process.

Let $\pi_{k_{v}, k_{d}}$ denote the state probability of a cell at statistical equilibrium. $\pi_{k_{v}, k_{d}}$ can je obtained from the state-transition rate diagram via matrix inversion subject to

$$
\sum_{\substack{0 \leq k_{v}+k_{d} \leq K_{u} \\ k_{d}=b, 2 b, \cdots}} \pi_{k_{v}, k_{d}}=1 .
$$

Let $\pi_{m_{v}, m_{d}}$ denote the steady state probability of $\left(m_{v}, m_{d}\right)$. Obviously, $\pi_{m_{v}, m_{d}}$ can be obtained from $\pi_{k_{v}, k_{d}}$ via the relation $m_{d}=k_{d} / b$.

We use $B_{v}$ and $B_{d}$ to denote the blocking probability of a voice and data call, respectively. For each type, we further divide it into two categories : new and handoff. $B_{v, n}, B_{v, h}, B_{d, n}, B_{d, h}$ are used for such purpose. For example $B_{v, h}$ denotes the blocking probability when the call is a handoff voice.

In this paper we use the following strategy to determine whether to admit a call. New calls are admissible only when $k_{v}+k_{d}+x \leq K_{l}$ where $x$ denotes the bandwidth required in accepting the new call, i.e. $x=1$ or $b$. Handoff calls are admissible as long as $k_{v}+k_{d}+x \leq K_{u}$.

Following the above strategy, various blocking probabilities can be obtained as follows.

$$
\begin{aligned}
B_{v, n} & =\sum_{k_{v}+k_{d} \geq K_{l}} \pi_{k_{v}, k_{d}} \\
B_{v, h} & =\sum_{k_{v}+k_{d}=K_{v}} \pi_{k_{v}, k_{d}} \\
B_{d, n} & =\sum_{K_{\imath}-b+1 \leq k_{v}+k_{d} \leq K_{v}} \pi_{k_{v}, k_{d}} \\
B_{d, h} & =\sum_{K_{u}-b+1 \leq k_{v}+k_{d} \leq K_{v}} \pi_{k_{v}, k_{d}}
\end{aligned}
$$

Another measure of interest is channel utilization. Let $\eta$ denote the average number of busy channels in 
a cell. Similar to the break-up of B into $B_{v}$ and $B_{d}$ we have $\eta_{v}$ and $\eta_{d}$ for $\eta$. Then

$$
\begin{aligned}
& \eta_{v}=\sum k_{v} \pi_{k_{v}, k_{d}} \\
& \eta_{d}=\sum k_{d} \pi_{k_{v}, k_{d}}
\end{aligned}
$$

\section{NUMERICAL EXAMPLES AND DISCUSSIONS}

In addition to the call admission policy treated in Sec. II, two other alternatives are considered for comparision. For convenience let us call the previous scheme soft admission (SA). The new schemes for comparision are sort of hard schemes. One of them works as follow. A call is always rejected whenever $k_{v}+k_{d}+x$ exceeds $K_{l}$ even if it were a handoff call. This scheme will be called hard admission (HA). The other scheme works exactly like hard admission except that handoff calls are given priority treatment by having certain amount of guard channels. We call this last scheme hard admission with guard channels (HAG). We shall use $n_{g}$ to denote the number of guard channels.

Fig. 1 plots handoff blocking probabilities $B_{v}$ and $B_{d}$ versus aggregate traffic intensity $\rho=\lambda / \mu=\left(\lambda_{v}+\right.$ $\left.\lambda_{d}\right) / \mu$ under $K_{u}=27, K_{l}=17, b=2, \beta=\lambda_{d} / \lambda=0.2$. Fig. 1(a) does it for $\gamma=\lambda_{v, h} / \lambda_{v, n}=\lambda_{d, h} / \lambda_{d, n}=0.2$ while $\gamma=0.4$ in Fig. 1(b). In Fig. $1, B_{v, h}$ and $B_{d, h}$ are represented by dotted and solid line, respectively. Three different values of $\xi, 0.0,0.3$ and 1.0 are considered for illustrative purpose. Because the soft admission strategy has the intention to accept more calls, we observe that SA offers considerably better handoff blocking probability although HAG does give handoff calls priority treatment. We also observe that $B_{d, h}$, blocking probability of data calls, appears to be higher than $B_{v, h}$. This is due to the fact that a data call consumes more bandwidth. Compare Fig. 1(a) and Fig. 1(b) we notice that due to the inclusion of more handoff traffics blocking probabilities $B_{v, h}$ and $B_{d, h}$ in Fig. 1(b) are higher. Larger $\gamma$ can be found in system with smaller cells, e.g. microcells.

Fig. 2 plots new call blocking probability for the same configuration considered in Fig. 1. Compare HA and HAG we observe that $H A$ offers better new call blocking probability for both voice and data calls than HAG since HA does not reserve channels solely for handoff calls. When $\rho$ is small, SA and HA have close performance in new call blocking probability. Fig. 3 shows channel utilization $\eta=\eta_{v}+\eta_{d}$ versus $\rho$. Also plotted in Fig. 3 is the number of busy users occupying the channels. We indeed observe that SA yields the highest utilization. Fig. 4 examines the effect of $b$ on blocking probabilities. We observe that both handoff and new call blocking probability are worsened by the increase in $b$ which is reasonable.

\section{CONCLUSIONS}

We have in this paper addressed the soft capacity aspect of a CDMA cellular system. Our major contribution is to obtain key performance measures such as blocking probability and channel utilization. The soft capacity aspect is reflected in a birth-and-death model. We have also conducted extensive numerical experiments using the results we derived and observed interesting but reasonable outcomes. Our results can be used in analyzing the performance of a practical system.

\section{REFERENCES}

[1] G. A. Arredondo, " AMPS - Advanced mobile phone service, " Bell Syst. Tech. J., Jan. 1979.

[2] D. J. Goodman, "Second Generation Wireless Information Networks, " IEEE Trans. Veh. Technol., vol. 40, pp. 366374, May 1991.

[3] Qualcomm, Mobile Station-Base Station Compatibility for Dual-Mode Wideband Spread Spectrum Cellular System, 1993.

[4] D. J. Goodman, P. S. Henry, and V.K. Prabhu, " Frequency-Hopped Multilevel FSK for Mobile Radio," Bell System Tech. J., vol. 59, pp. 1257-1275, Sep. 1980.

[5] W. C. Y. Ine, "Overview of Cellular CDMA, "IEEE Trans . Veh. Technol., Vol. 40, pp. 291-302, May 1991.

[6] K. S. Gilhousen, I. M. Jacobs, R. Padovani, A. J. Viterbi, L. A. Weaver and C. E. Wheatley," On the Capacity of a Cellular CDMA System," IEEE Trans. Veh. Technol., vol. 40, pp. 303-312. May 1991.

[7] R.L. Pickholtz, L. B. Milstein, and D.L. Schilling, "Spread Spectrum for Mobile Communications, " IEEE Trans. Veh. Technol, vol. 40, pp. 313-322. May 1991.

[8] L. B. Milstein, D. L. Schilling, R. L. Pickholtz, V. Erceg, M. Kullbeck, E. G. Kanterakis, D. S. Fishman, W. H. Biederman, and D. C. Salerno, "On the Feasibility of a CDMA Overlay for Personal Communication Networks," IEEE J. Sel. Areas in Commun., vol. 10, pp. 655-668, May 1992.

[9] Y. R. Tsai and J. F. Chang, "The Feasibility of Adding Personal Communication Networks to a Existing Fixed Service Microwave System, " Submitted.

[10] R. R. Gejij, "Forward-Link-Power Control in CDMA Cellular Systems, " IEEE Trans. Veh. Technol., vol. 41, pp. 532-536, Nov. 1992.

[11] K. Feher, Digital Communications Satellite/Earth Station Engineering, Chap. 4, Prentice-Hall, Inc., Englewood Cliffs, N.J., 1983.

[12] K. I. Kim, “ CDMA Cellular Engineering Issues, " IEEE Trans. Veh. Technol., vol. 42, pp. 345-350, Aug. 1993.

[13] M. S. Alencar, I. F. Blake, "The Capacity for a DiscreteState Code Division Multiple-Access Channel, " IEEE J. Sel. Areas in Commun., vol. 12 pp. 925-937, June 1994.

[14] C. H. Yoon and C. K. Un, "Performance of Personal Portable Radio Telephone Systems with and without Guard Channels, "IEEE J. Sel. Areas in Commun., vol. 11, pp. 911-917, Aug. 1993.

[15] R. A. Guerin, "Channel Occupancy Time Distribution in a Cellular Radio System, " IEEE Trans. Veh. Technol., vol. 35, pp. 89-99, Aug. 1987. 

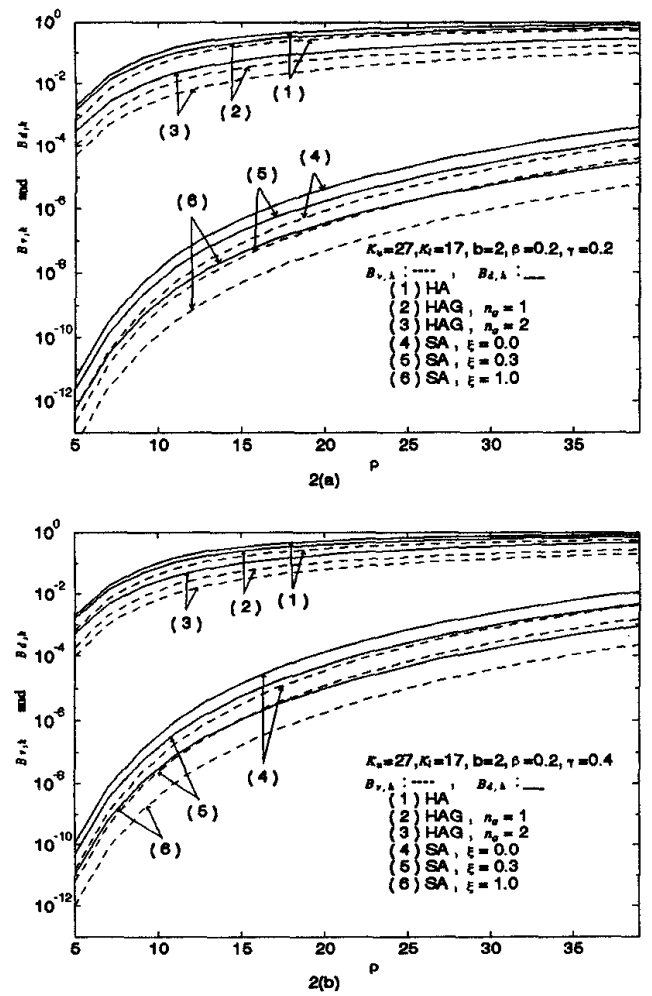

Fig. 1 Handoff blocking probability $B_{v, h}$ and $B_{d, h}$ versus traffic intensity $\rho$ (a) $\gamma=0.2$ (b) $\gamma=0.4$
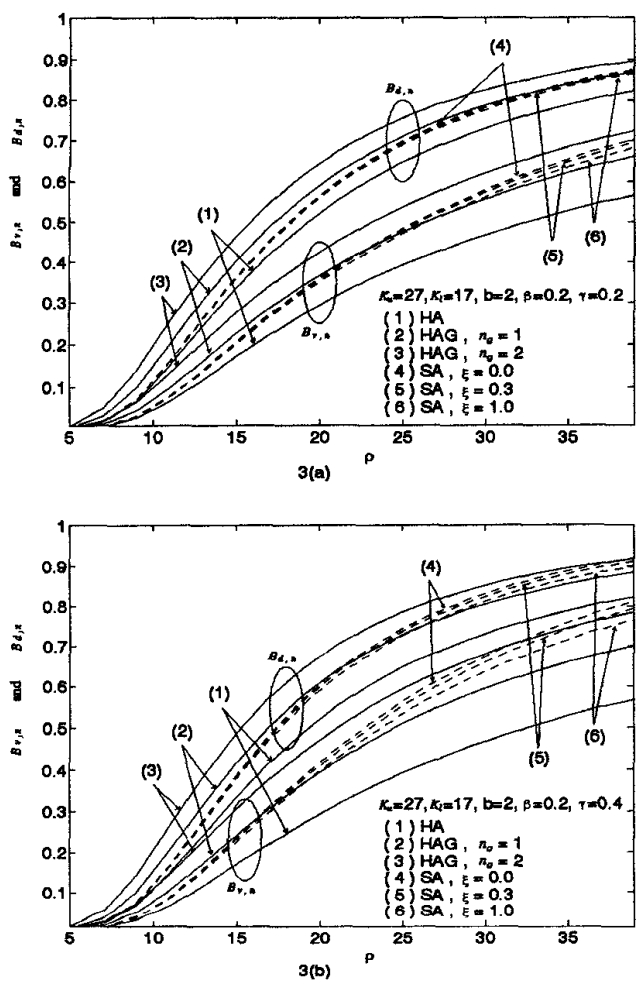

Fig. 2 New call blocking probability $B_{v, n}$ and $B_{d, n}$ versus traffic intensity $\rho$ (a) $\gamma=0.2$ (b) $\gamma=0.4$
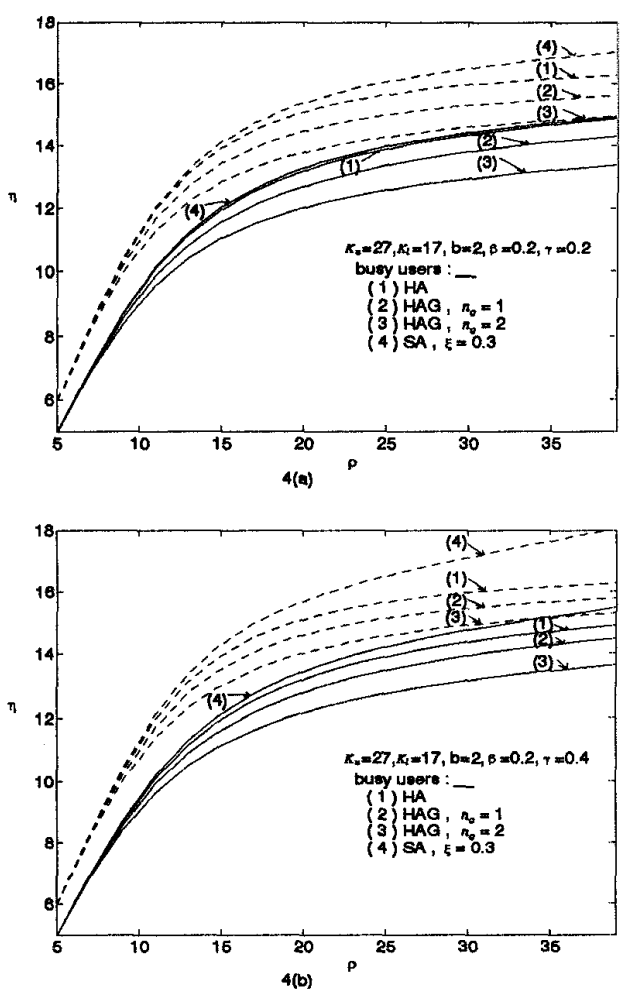

Fig. 3 Channel utilization versus traffic intensity $\rho$ (a) $\gamma=0.2$ (b) $\gamma=0.4$
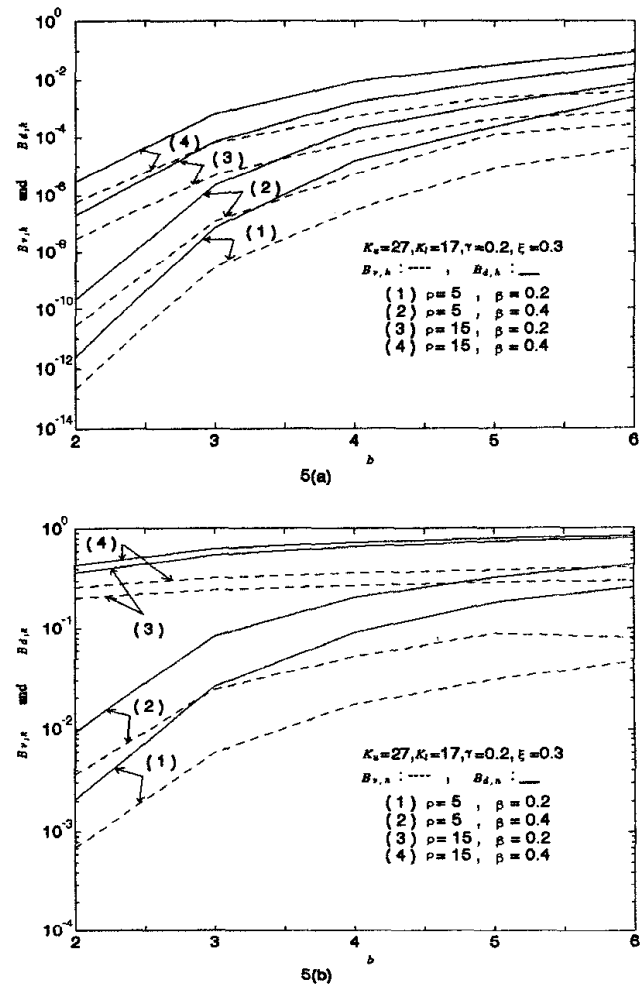

Fig. 4 Blocking probability versus b (a) $B_{v, h}$ and $B_{d, h}$ (b) $B_{v, n}$ and $B_{d, n}$ 